\title{
Resultados de la cirugía de reconstrucción esofágica en pacientes pediátricos con patología esofágica compleja en dos hospitales de alto nivel de Medellín, Colombia, 2006-2016
}

\author{
Natalia Herrera-Toro', María Elena Arango-Rave², Laura Peña-Aguirre³ ${ }^{3}$, Michelle Carolina Silvera-Valera ${ }^{4}$
}

\section{RESUMEN}

Objetivos: el esófago difícil (complejo) representa la vía final común de un conjunto de enfermedades que requieren múltiples procedimientos o cirugía de reemplazo esofágico; ya sea porque desde el inicio es la única alternativa de manejo o porque han fallado previamente otros tipos de intervenciones menos radicales. El objetivo de este estudio es describir los resultados de la cirugía de reconstrucción esofágica en pacientes con patología esofágica compleja en dos hospitales de alto nivel de Medellín (Colombia) entre el $1^{\circ}$ enero de 2006 y el 31 de junio de 2016.

Métodos: estudio descriptivo retrospectivo en el que se revisaron las historias clínicas del Hospital Pablo Tobón Uribe (HPTU) y el Hospital Universitario San Vicente Fundación (HSVF), recopilando 40 pacientes que cumplieron con los criterios de inclusión.

Resultados: el diagnóstico más frecuente de esófago difícil corresponde a atresia de esófago, seguido de quemadura esofágica. Las causas directas de la condición fueron estenosis esofágicas persistentes, fístulas traqueoesofágicas recurrentes, fístulas esofagopleurales y brecha larga. Las intervenciones quirúrgicas fueron en orden de frecuencia: ascenso gástrico, corrección de la fístula, interposición de colon, esofagostomía más gastrostomía, resección, anastomosis y esofagoplastias. Las principales complicaciones posoperatorias fueron la estenosis,

1 Médica general de la Universidad de Antioquia, cirujana general de la Universidad de Antioquia, cirujana infantil de la Universidad de Antioquia, Cirujana pediátrica del hospital Pablo Tobón Uribe. Profesora de la Universidad de Antioquia, Medellín, Colombia.

2 Médica, cirujana general y cirujana infantil de la Universidad de Antioquia. Cirujana pediátrica del hospital San Vicente Fundación. Profesora de la Universidad de Antioquia.

3 Médica y cirujana de la Universidad de Antioquia, Medellín, Colombia. Médica rural en Hospital de Fredonia, Antioquia.

4 Médica y cirujana de la Universidad Pontificia Bolivariana. Médica general en el Hospital La María, Medellín, Colombia.

Correspondencia: Natalia Herrera Toro; nataherrerat@gmail.com

Recibido: febrero 01 de 2017

Aceptado: mayo 3 de 2017

Cómo citar: Herrera-Toro N, Arango-Rave ME, Peña-Aguirre L, Silvera-Valera MC. Resultados de la cirugía de reconstrucción esofágica en pacientes pediátricos con patología esofágica compleja en dos hospitales de alto nivel de Medellín, Colombia, 2006-2016. latreia. 2017 0ct-Dic;30(4): 369-375. D0I 10.17533/udea.iatreia.v30n4a02. 
las fístulas, la infección del sitio operatorio y los trastornos funcionales.

Conclusiones: los niños con patología esofágica compleja representan un reto para los cirujanos pediátricos, tanto por la dificultad de los procedimientos a los que son sometidos como por la morbilidad de las enfermedades que conllevan y de las cirugías que requieren para dar continuidad al tracto digestivo.

\section{PALABRAS CLAVE}

Atresia Esofáoica; Cáusticos; Enfermedades Del Esófago; Esófago

\section{SUMMARY}

Esophageal reconstruction results in surgery of pediatric patients with complex esophageal pathology in two high level hospitals of Medellín, Colombia, 2006-2016

Introduction: complex esophagus represents a final condition that occurs secondary to diseases that require multiple procedures or esophageal replacement surgery, either because it is the only treatment or because others interventions have failed. Our objective is to describe the results of esophageal reconstruction surgery in patients with complex esophageal pathology attended in two high-level hospitals in Medellin, Colombia, between January 1- 2006 and June 31- 2016.

Methods: descriptive and retrospective study with review of clinical records at Hospital Pablo Tobón Uribe (HPTU) and Hospital Universitario San Vicente Fundación (HSVF), collecting 40 patients.

Results: the most frequent diagnosis was esophageal atresia, followed by esophageal burn. The main causes that led these patients to be classified as complex esophagus were persistent esophageal strictures, recurrent tracheoesophageal fistulas, esophageal fistulas and the long gap. The surgical interventions were in order of frequency: gastric replacement, closure of the fistula, colon replacement, esophagostomy and gastrostomy, resection and anastomosis and esophagoplasty. The main postoperative complications of patients undergoing esophageal reinterventions or esophageal replacement were stenosis, fistulas, surgical site infection and functional disorders.
Conclusions: children with complex esophageal pathology represent a challenge for pediatric surgeons, because of the difficulty of the procedures they undergo as well as the morbidity of their diseases and surgeries they require to give continuity to their digestive tract.

\section{KEY WORDS}

Caustics; Esophageal Atresia; Esophagus; Esophagus Diseases

\section{RESUMO}

Resultados da cirurgia de reconstrução esofágica em pacientes pediátricos com patologia esofágica complexa em dois hospitais de alto nível de Medellín, Colômbia, 2006-2016

As crianças com patologia esofágica complexa representam um desafio para os cirurgiões pediátricos, tanto pela dificuldade dos procedimentos aos que são submetidos, como pela morbidez das doenças que implicam e das cirurgias que requerem para dar continuidade ao trato digestivo. O objetivo do estudo é descrever os resultados da cirurgia de reconstrução esofágica em pacientes com patologia esofágica complexa, definida como a falha no manejo endoscópico ou na cirurgia de reparação inicial em dois hospitais de alto nível de Medellín, Colômbia entre o dia $1^{\circ}$ janeiro de 2006 e o dia 31 de junho de 2016. Se revisaram as histórias do Hospital Pablo Tobón Uribe (HPTU) e Hospital San Vicente Fundación (HSVF), recopilando 40 pacientes. O diagnóstico mais frequente de esôfago difícil corresponde a atresia de esôfago, seguido de queimadura esofágica. As principais causas que levaram a estes pacientes a catalogar-se como esôfago complexo foram: estenose esofágicas persistentes e fístulas traqueoesofágicas recorrentes, fístulas esôfago-pleurais e brecha longa. As intervenções cirúrogicas foram em ordem de frequência: Ascenso gástrico, correção da fístula, interposição de colón, esofagostomia mais gastrostomia, ressecção, anastomose e esofagoplastias. Apresentam-se as principais complicações pós-operatórias e o tempo de estância hospitalar, nutrição parenteral e em reiniciar a via oral, ademais, mostramos o seguimento pós-cirúrogico. 


\section{PALAVRAS CHAVE}

Atresia Esofáóica; Esôfago; Cáusticos; Doenças Do Esôfago

\section{INTRODUCCIÓN}

La reconstrucción esofágica es el resultado de procedimientos quirúrogicos que tienen como finalidad restaurar la anatomía y la funcionalidad del tracto gastrointestinal en niños con atresia de esófago de brecha larga, estenosis por quemaduras cáusticas, secuelas de cirugías previas o problemas funcionales. Considerando que las enfermedades esofágicas complejas tienen gran impacto en la sobrevida y en la calidad de vida de la población pediátrica, existe un permanente debate en cuanto a las técnicas quirúroicas más adecuadas para el tratamiento de estos pacientes, que generalmente implican múltiples procedimientos esofágicos, o incluso el remplazo del esófago. Dado que en Medellín (Colombia), no contamos con información objetiva sobre los resultados de las diferentes técnicas quirúrogicas a las que son sometidos los niños con diagnóstico de patologías esofágicas complejas, decidimos recopilar esta información en dos de los principales hospitales de la ciudad, con el fin de crear nuestras propias estadísticas.

\section{MATERIALES Y MÉTODOS}

Objetivo: describir los resultados de la cirugía de reconstrucción esofágica en pacientes con diagnóstico de patología esofágica compleja definida por la falla en el manejo endoscópico o en la cirugía de reparación inicial, en dos hospitales de alto nivel de la ciudad de Medellín, Colombia.

Tipo de estudio: estudio observacional, descriptivo, retrospectivo de una serie de casos.

población y muestra: se revisaron las historias clínicas de los pacientes menores de 15 años quienes ingresaron al servicio de cirugía infantil del HPTU y del HUSVF, con patologías esofágicas complejas congénitas y adquiridas entre el $1^{\circ}$ de enero de 2006 y el 31 de junio del 2016.

En la revisión de las historias clínicas se seleccionaron todos los pacientes con diagnóstico de enfermedad esofágica por los códigos del CIE 10 (K219, K220, K221, K222, K223, K225, K228, K229, K238, Q390, Q391, Q392, Q393, Q395, Q396, Q398, Q399, T181, T281, T286, S112) y en la revisión de todas las historias, se incluyeron los que habían sido llevados a procedimientos quirúrgicos.

Se diseñó una base de datos en el programa Excel con las reglas de validación pertinentes, garantizando la calidad de los datos. Se solicitó autorización en los comités de investigaciones y ética de ambas instituciones hospitalarias y se realizó el curso de buenas prácticas clínicas del HPTU, como requisitos para llevar a cabo la investigación.

Se definió el peso adecuado para la edad según el programa de la OMS, Anthro (para menores de 5 años) y Anthro plus (mayores de 5 años), como base para determinar si existía algún grado de desnutrición en el seguimiento de los pacientes.

\section{RESULTADOS}

Se revisaron las historias de los últimos 10 años del HPTU y 5 años del HSVF, recopilando 40 pacientes que cumplen con los criterios de inclusión. Los datos demográficos de los 40 pacientes se encuentran en la tabla No. 1.

El diagnóstico inicial más frecuentemente encontrado dentro de los pacientes que se catalogaron como esófago difícil, correspondió a la atresia de esófago en un total de 24 pacientes (60\%), siendo la atresia tipo III la más común en 15 pacientes, seguida de la tipo I en 7 pacientes y la tipo II en 2 pacientes. El segundo diagnóstico en orden de frecuencia correspondió a la quemadura esofágica en 8 pacientes (20\%), seguida por otros diagnósticos en 8 pacientes restantes (20\%) entre los cuales se encuentran la acalasia, la necrosis esofágica posoperatoria, las fístulas traqueoesofágicas adquiridas y las estenosis congénitas y adquiridas del esófago.

Las causas que llevaron a estos pacientes a diagnosticarse como esófago complejo fueron en orden de frecuencia: estenosis esofágicas persistentes (14 pacientes, 35\%); fístulas traqueoesofágicas recurrentes (10 pacientes, 24\%); fístulas esofagopleurales inmanejables (7 pacientes, 17\%) y la atresia esofágica de brecha larga con necesidad sustitución esofágica primaria (9 pacientes, 22,5\%). 
Tabla 1. Características demográficas de los pacientes

\begin{tabular}{|lcc|}
\hline & n & (\%) \\
\hline Número de pacientes & 40 & \\
\hline Sexo femenino & 18 & 45 \\
\hline Sexo masculino & 22 & 55 \\
\hline Comorbilidades & & \\
\hline Cardiovasculares & 11 & 27 \\
\hline Respiratorias & 10 & 25 \\
\hline Perinatales & 9 & 22,5 \\
\hline SNC & 8 & 20 \\
\hline Genéticas & 8 & 20 \\
\hline Gastrointestinales & 8 & 20 \\
\hline Ninguna & 10 & 25 \\
\hline Otras & 11 & 27,5 \\
\hline Diagnóstico inicial & & \\
\hline Atresia esofágica & 24 & 60 \\
\hline Quemadura esofágica & 8 & 20 \\
\hline Otro & 8 & 20 \\
\hline SN: Sistema Nervioso Centrat & & \\
\hline
\end{tabular}

SNC: Sistema Nervioso Central.

Dentro de los procedimientos utilizados para el manejo de la patología esofágica compleja en ambos hospitales, se encontró que únicamente 2 pacientes fueron sometidos a tratamiento endoscópico con sellante de fibrina para el cierre de fístula traqueoesofágica y en ninguno de los casos se obtuvo un resultado exitoso.

Las intervenciones quirúroicas realizadas en los pacientes catalogados con patología esofágica compleja comprendieron: ascenso gástrico en 17 pacientes $(42,5 \%)$, cierre quirúrogico de fístula en 8 pacientes (20\%), interposición de colon en 5 pacientes (12,5\%), esofagostomía más gastrostomía en 4 pacientes (10\%), resección $y$ anastomosis del segmento esofágico comprometido en 3 pacientes $(7,5 \%)$ y plastias del esófago en un paciente $(2,5 \%)$.

Las complicaciones globales de todos los pacientes sometidos a reintervenciones esofágicas y a remplazo esofágico fueron: estenosis esofágica en 24 pacientes (60\%), fístulas esofagocutáneas en 10 pacientes Ilevados a reemplazo esofágico (25\%), fístulas esofagopleurales en 6 pacientes (15\%), infecciones del sitio operatorio en 4 pacientes $(10 \%)$ y trastornos funcionales en 8 pacientes (20\%). Otras complicaciones menos frecuentes incluyeron hernias incisionales, fístula traqueoesofágica recurrente y dehiscencia completa en uno de los casos.

Con respecto a la mortalidad, se presentaron tres muertes, una de ellas relacionada con el procedimiento quirúrgico $(2,5 \%)$ y las otras 2 a causa de situaciones ajenas a la intervención de la patología esofágica compleja (5\%).

Cuando evaluamos los resultados, sólo en los casos de remplazo esofágico, encontramos que tres de los niños sometidos a ascenso gástrico no presentaron complicaciones, incluyendo uno de ellos que fue llevado a ascenso primario el primer día de vida. Como complicaciones posoperatorias se presentaron 14 casos de estenosis $(82,3 \%$ ) y 7 casos de fístulas 
Tabla 2. Resultados

\begin{tabular}{lcccc}
\hline & $\begin{array}{c}\text { Tiempo mínimo } \\
\text { (días) }\end{array}$ & $\begin{array}{c}\text { Tiempo máximo } \\
\text { (días) }\end{array}$ & $\begin{array}{c}\text { Media de tiempo } \\
\text { (días) }\end{array}$ & $\begin{array}{c}\text { Mediana de } \\
\text { tiempo (días) }\end{array}$ \\
\hline Tiempo para inicio de vía oral & 3 & 74 & 18,4 & 10,5 \\
Tiempo de NPT & 0 & 200 & 19,97 & 11 \\
Estancia en UCIP y UCEP & 1 & 116 & 13,6 & 10 \\
Estancia hospitalaria & 6 & 134 & 34,69 & 27 \\
\hline
\end{tabular}

NPT: nutrición parenteraltotal. UCIP: unidad de cuidados intensivos pediátrica. UCEP: unidad de cuidados especiales pediátrica

esofagocutáneas (41,1\%). Se pudo concluir que todos los niños que desarrollaron fístula esofágica cervical presentaron estenosis posterior.

En cuanto a la interposición de colon, 3 de los pacientes en quienes se practicó dicha intervención presentaron estenosis cervical (60\%) y 2 de ellos tuvieron previamente fístula esofagocutánea. Un paciente de este grupo no presentó ningún tipo de complicación.

Ningún niño operado de remplazo esofágico presentó muerte relacionada con la cirugía.

Los tiempos de inicio de vía oral, duración de NPT (Nutrición parenteral total), estancia hospitalaria total y estancia en cuidado crítico (UCI y UCE pediátrica) se muestran en la tabla No. 2

Cuando revisamos en las historias clínicas el seguimiento posoperatorio realizado por la consulta externa de cirugía pediátrica o de gastroenterología pediátrica, después del alta hospitalaria, encontramos los siguientes datos: dicho seguimiento fue en promedio de 21.61 meses, se presentó una pérdida de 6 pacientes en el seguimiento; de los 34 pacientes restantes, se encontró que 27 de ellos lograron alimentación por vía oral exclusiva (67,5\%), 2 se alimentan por vía oral y gastrostomía (5\%), uno por vía oral y sonda nasoenteral (2,5\%), uno por sonda nasogástrica $(2,5 \%)$ y 3 por sonda de gastrostomía $(7,5 \%)$. Nueve de los pacientes presentaron disfagia $(26,4 \%)$ y 11 reflujo gástrico (32,3\%); ninguno requirió nutrición parenteral total ambulatoria. Diez pacientes tenían peso adecuado para la edad $(29,4 \%)$, el resto de ellos presentaron bajo peso para la edad de acuerdo a los percentiles de normalidad determinados por la OMS.

\section{DISCUSIÓN}

Entendemos el término esófago difícil como la patología esofágica que se produce a causa de un conjunto de enfermedades que requieren de múltiples procedimientos o de cirugía de reemplazo esofágico, ya sea porque desde el inicio representa la única alternativa de manejo, o porque han fallado previamente otros tipos de intervenciones menos radicales (quirúroicas y endoscópicas). Entre las principales patologías reportadas en la literatura, que por su naturaleza o por las complicaciones derivadas de su tratamiento conllevan al diagnóstico de esófago difícil encontramos, en su orden, la atresia esofágica y las estenosis esofágicas por cáusticos y otras causas como postinflamatorias, secundarias a reflujo gastroesofágico (hasta en el 15\% de los casos), infecciosas (candidiasis $y$ herpes), postirradiación (poco frecuentes en niños), congénitas e incluso tras procedimientos quirúrogicos para corrección de atresia esofágica (8 a 79\%) (1-4). En nuestra serie de casos también encontramos la atresia de esófago como la patología predominante, seguida por las quemaduras esofágicas por cáusticos y las estenosis pépticas y congénitas, entre otras causas. Adicional a estos diagnósticos, la acalasia tuvo un papel importante, clasificándose como patología esofágica compleja según la evolución en su tratamiento.

En nuestra revisión, las causas más frecuentes de esófago difícil fueron las mismas reportadas en la literatura (estenosis, dehiscencias y fístulas recurrentes) (5). La comparación entre nuestros resultados posoperatorios y otros publicados a nivel mundial, se presenta en la tabla 3.

Para el manejo de las fístulas esofágicas recurrentes se encuentran diferentes técnicas, entre éstas 
el tratamiento endoscópico usando sellante de fibrina, que se ha mostrado como alternativa a la toracotomía y al reparo quirúrgico (8). Otras técnicas implementan el uso de sellantes asociado a diatermia, mostrando reducción en la morbilidad $y$ recurrencia (9); resultados que se evidencian también en publicaciones retrospectivas encontradas en la literatura (10). Esta revisión evidenció el poco uso de esta opción en nuestro medio y probablemente la limitada experiencia influyó en que los únicos dos pacientes en los que se aplicó dicha técnica, no se presentaran resultados exitosos.

Algunos autores han encontrado que la calidad de vida de los niños sometidos a cirugías complejas del esófago es más favorable cuando se preserva el esófago nativo efectuando plastias, resecciones parciales y dilataciones, que cuando se hacen remplazos esofágicos (11). En los casos en los que la preservación del esófago no es posible, se debe proceder con cirugías de remplazo esofágico. Entre las décadas de los años 70 y los 80 surgieron nuevas alternativas, empleando el estómago al crear un tubo gástrico descrito por Ein y la transposición gástrica descrita por Spitz y Atwell, y otras mediante la interposición del colon inicialmente descrita por Lundblad en 1921 (12).

El pronóstico de la cirugía de remplazo esofágico es favorable, encontrando reportes hasta de 90\% de satisfacción en el seguimiento (13). En nuestro estudio encontramos que, para el remplazo esofágico, utilizamos con más frecuencia el ascenso gástrico que la interposición colónica y que las complicaciones más frecuentemente asociadas a estas cirugías corresponden a la aparición de fístula esofagocutánea cervical en el sitio de la anastomosis, con cifras altas comparadas con otros estudios, y también a la estenosis posterior, la cual responde

Tabla 3. Resultados en el manejo quirúrgico de patología esofágica

\begin{tabular}{lccc}
\hline \multicolumn{1}{c}{ Estudio } & Estenosis & Fístula esofagocutánea o salivar & Mortalidad \\
\hline Herrera, Arango, Peña, Silvera & $37 \%$ & $10 \%$ & $7,5 \%$ \\
\hline Spitz, Kiely, Pierro.* (6) & $19,6 \%$ & $12 \%$ & $5,2 \%$ \\
\hline Engum, Grosfeld, West, Rescorla, Scherer.(5) & $35 \%$ & $16 \%$ & $5 \%$ \\
\hline Ávila, Encina, Suárez, Martínez, Fernández, Queizán y col. (1) & $10,3 \%$ & $20,7 \%$ & $3,5 \%$ \\
\hline Koivusalo, Pakarinem, Rintala. (7) & $78 \%$ & - & $2 \%$ \\
\hline
\end{tabular}

*Sólo ascensos gástricos

de forma adecuada a las dilataciones. Además, se halló una asociación importante entre estas fístulas y la estenosis, evidenciando que todos los pacientes que presentaron fístula de la anastomosis esofágica posterior a la sustitución del esófago con ascenso gástrico, desarrollaron estenosis.

De la mortalidad de nuestros niños que fue del 7,5\%, el $2,5 \%$ estuvo relacionada con el procedimiento quirúrgico y sus complicaciones. Otros estudios presentan cifras de mortalidad del 12,6\% (14), 14\% (15) y $5,2 \%$ en publicaciones tan grandes como las de Spitz y col. (6).

En el seguimiento de nuestros pacientes pudimos corroborar que un porcentaje importante de ellos reciben alimentación por vía oral, $88 \%$, lo que representa un gran avance en la mejoría de la calidad de vida de los niños con patologías esofáoicas complejas. Además, el 29\% tienen peso adecuado para su edad. Algunos autores, por ejemplo, con el ascenso gástrico, han encontrado que todos sus 
pacientes tienen peso bajo en el seguimiento (16), y otros reportan el $41 \%$ de bajo peso para la edad (14).

\section{LIMITACIONES DEL ESTUDIO}

Es un estudio que incluye variadas patologías esofágicas por lo tanto puede suceder que no se obtengan resultados finales individualizados sino de las entidades que se incluyeron de forma global.

En el momento de la revisión de historias clínicas, se evidenció que algunas de las más antiguas se encontraban en programas anteriores a los usados en la actualidad tanto en el HUSVF como con el HPTU, por lo tanto, determinada información no estuvo disponible. Por esta razón, se pudieron haber perdido datos sobre algunos de los niños que fueron incluidos en el estudio o incluso, perder pacientes que cumplían con los criterios de inclusión.

También hubo limitaciones en el seguimiento, ya que, por razones externas, en ciertos pacientes continuaron sus revisiones en otros hospitales y se perdió su evolución.

\section{CONCLUSIONES}

- En nuestro medio, encontramos que la principal patología esofáogica relacionada con el diaonnóstico de esófago difícil fue la atresia esofágica.

- El procedimiento quirúrgico más usado para el remplazo esofágico fue el ascenso gástrico.

- Respecto a los resultados posoperatorios, la principal complicación del remplazo esofáogico fue la estenosis.

- En nuestra serie se presentó mayor tasa de fístulas esofagocutáneas comparado con los otros reportes de la literatura mundial. Además, todos los niños que presentaron fístula cervical desarrollaron estenosis posterior.

- En nuestras instituciones no tenemos adecuada experiencia con el manejo endoscópico para el tratamiento de las fístulas traqueoesofágicas.

- El promedio de seguimiento de los pacientes en nuestro medio es inferior al reportado en otros estudios, $y$ en dicho seguimiento encontramos que un alto porcentaje de nuestros pacientes logró la alimentación por vía oral y el 29\% de ellos presentan un peso adecuado para la edad.

\section{CONFLICTOS DE INTERESES}

Ninguno por declarar.

\section{REFERENCIAS BIBLIOGRÁFICAS}

1. Ávila LF, Luis AL, Encinas JL, Andrés AM, Suárez O, Martínez L, et al. Sustitución esofágica. Experiencia de 12 años. Cir Pediatr. 2006 Oct;19(4):217-22.

2. Pearson EG, Downey EC, Barnhart DC, Scaife ER, Rollins MD, Black RE, et al. Reflux esophageal stricture--a review of 30 years' experience in children. J Pediatr Surg. 2010 Dec;45(12):2356-60. DOI 10.1016/j. jpedsurg. 2010.08.033.

3. Romero Manteola E, Ravetta P, Paredes E, Defagó $V$, Riga C. Estenosis esofágica congénita: ¿Dilatación o ciruogía? Rev Pediatr Electrón. 2009;6(3).

4. Tan Y, Zhang J, Zhou J, Duan T, Liu D. Endoscopic Incision for the Treatment of Refractory Esophageal Anastomotic Strictures in Children. J Pediatr Gastroenterol Nutr. 2015 Sep;61(3):319-22. DOI 10.1097/ MPG.0000000000000801.

5. Engum SA, Grosfeld JL, West KW, Rescorla FJ, Scherer LR 3rd. Analysis of morbidity and mortality in 227 cases of esophageal atresia and/or tracheoesophageal fistula over two decades. Arch Surg. 1995 May;130(5):502-8; discussion 508-9.

6. Spitz L, Kiely E, Pierro A. Gastric transposition in children--a 21-year experience. J Pediatr Surg. 2004 Mar;39(3):276-81; discussion 276-81.

7. Koivusalo AI, Pakarinen MP, Rintala RJ. Modern outcomes of oesophageal atresia: single centre experience over the last twenty years. J Pediatr Surg. 2013 Feb;48(2):297-303. DOI 10.1016/j.jpedsurg. 2012.11.007.

8. Meier JD, Sulman CG, Almond PS, Holinger LD. Endoscopic management of recurrent congenital tracheoesophageal fistula: a review of techniques and results. Int J Pediatr Otorhinolaryngol. 2007 May;71(5):691-7.

9. Richter GT, Ryckman F, Brown RL, Rutter MJ. Endoscopic management of recurrent tracheoesophageal 
fistula. J Pediatr Surg. 2008 Jan;43(1):238-45. DOI 10.1016/j.jpedsurg. 2007.08.062.

10. Gutiérrez San Román C, Barrios JE, Lluna J, Ibañez V, Hernández E, Ayuso L, et al. Long-term assessment of the treatment of recurrent tracheoesophageal fistula with fibrin glue associated with diathermy. J Pediatr Surg. 2006 Nov;41(11):1870-3.

11. Dingemann C, Meyer A, Kircher G, Boemers TM, Vaske B, Till $\mathrm{H}$, et al. Long-term health-related quality of life after complex and/or complicated esophageal atresia in adults and children registered in a German patient support group. J Pediatr Suro. 2014 Apr;49(4):631-8. DOI 10.1016/j.jpedsurg. 2013.11.068.

12. Spitz L, Coran A. Esophageal replacement. In: Pediatric Surgery. $7^{\text {th }}$ ed. Ámsterdam: Elsevier; 2012. p. 92738. DOI 10.1016/B978-0-323-07255-7.00071-4.
13. Spitz L. Esophageal replacement: overcoming the need. J Pediatr Surg. 2014 Jun;49(6):849-52. DOI 10.1016/j.jpedsurg.2014.01.011.

14. Lacher M, Froehlich S, von Schweinitz D, Dietz HG. Early and long term outcome in children with esophageal atresia treated over the last 22 years. Klin Padiatr. 2010 Sep;222(5):296-301. DOI 10.1055/s-00301249610 .

15. Gupta DK, Sharma S, Arora MK, Agarwal G, Gupta $M$. Grover VP. Esophageal replacement in the neonatal period in infants with esophageal atresia and tracheoesophageal fistula. J Pediatr Surg. 2007 Sep;42(9):1471-7.

16. Jain $\nabla$, Sharma S, Kumar R, Kabra SK, Bhatia V, Gupta DK. Transposed intrathoracic stomach: functional evaluation. Afr J Paediatr Surg. 2012 SepDec;9(3):210-6. DOI 10.4103/0189-6725.104722. 